\title{
Weak Interactions Effect on the P-N Mass Splitting and the Principle Of Equivalence
}

Cite as: AIP Conference Proceedings 623, 309 (2002); https://doi.org/10.1063/1.1489768

Published Online: 19 June 2002

N. Chamoun and H. Vucetich

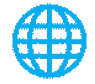

Challenge us.

What are your needs for periodic signal detection?

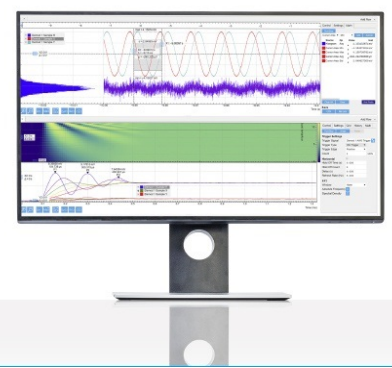

- Zurich

- Instruments 


\title{
Weak Interactions Effect on the P-N Mass Splitting and the Principle Of Equivalence
}

\author{
N. Chamoun* and H. Vucetich ${ }^{1 \uparrow}$ \\ ${ }^{*}$ Department of Physics, Higher Institute for Applied Sciences and Technology, Box 31983, \\ Damascus, Syria. \\ ${ }^{\dagger}$ IFUNAM, UNAM, México
}

\begin{abstract}
The neutron-proton mass difference is computed from a model-indepentent sum rule. When this contribution is included in the analysis of the Eötvös experiment, the bound for possible weak interactions violations to the equivalence principle is improved by one order of magnitude from $10^{-2}$ to $10^{-3}$.
\end{abstract}

The Principle of Equivalence (EP) is the physical basis of General Relativity. It loosely states that any freely falling reference frame is locally equivalent to an inertial reference frame [1]. This is a very strong statement: its unrestricted validity leads to General Relativity as the unique theory for the gravitational field [3] and experimental tests of its consequences probe deeply the structure of gravitation.

One of the consequences of EP is the Universality of Free Fall (UFF) which states that the world line of a body submerged in a gravitational field is independent of its composition and structure [2]. UFF, among the consequences of EP, is one of the strongest tests of its validity. For instance, it has been shown that sufficiently sensitive related experiments can provide strict tests on superstring theories (see, eg. [4]) or Kaluza-Klein theories (eg. [5]), thus exhibiting the presence of "new physics". Indeed, the STEP satellite experiment [6] will improve these tests sensitivity by many orders of magnitude.

One of the profound consequences of UFF is that all forms of non-gravitational energy should couple in the same way to the gravitational field. Any violation of UFF should break equation $m_{I}=m_{P}$ and the difference between inertial $m_{I}$ and passive gravitational mass $m_{P}$ of a body could be expressed via phenomenological parameters $\Gamma_{i}$ specific to each form of interaction and reflecting its degree of violation to the equivalence principle:

$$
m_{I}-m_{P}=\Delta m=\sum_{i} \Gamma_{i} E_{i}
$$

where the binding energies $E_{i}$ are usually estimated with the semiempirical mass formula [9] or, in the case of weak interactions, a suitable generalization [10,11].

\footnotetext{
${ }^{1}$ On leave of absence, Observatorio Astronómico, Universidad Nacional de La Plata, Paseo del Bosque S/N, CP 1900 La Plata, Argentina.
} 
Eötvös experiments $[2,7,8]$ set an upper limit on the difference of acceleration in a gravitational field for different materials and so impose an upper bound on the violation parameters $\Gamma_{i}$. The parameter $\Gamma_{W}$, measuring the degree of violations of weak interactions, has a quite large bound $\left(10^{-2}\right)$, not only due to the tiny contribution of weak interactions to the total mass but also largely because the binding energy per nucleon due to weak interactions is a very slowly varying function across the periodic table which then leads to a large cancellation in the analysis of Eötvös experiments $[10,11]$. In order to improve the significance of Eötvös experiments with respect to weak interactions, one should include the individual nucleons contribution to the nucleus mass since it changes much faster along the periodic table.

There is a model-independent approach to the weak contribution to the proton-neutron mass difference: the development of a sum rule that gives the nucleon self mass in terms of observable quantities. This was first done for the electromagnetic interactions in [12] and will be called the generalized Cottingham formula. This sum rule (which, by the way, shows that the electromagnetic and weak contributions to the nucleon self-mass are finite in the Born approximation) is a rigorous model-independent way for computing the proton-neutron mass difference. Besides, it has been generalized to strong interactions [13]. Detailed proofs can be found in the above references.

We shall develop a sum rule corresponding to the weak interactions similar to Cottingham's formula. Because of weak isospin symmetry neither charged currents nor the axial part of the neutral current will contribute to the neutron-proton mass difference and only the vector neutral current will give a nonzero contribution for the difference. This current, however, has the same structure as the electromagnetic current and so the assumptions involved in the derivation of Cottingham's formula are still valid and following the steps in its derivation one gets the similar result:

To first order in the Fermi constant, the neutral current contribution to the self-energy of the nucleon may be written as:

$$
\Delta M_{N}^{e m}=\frac{i G_{F}}{2(2 \pi)^{4}} \int d^{4} q G_{Z}^{\mu \nu}\left(q^{2}\right) T_{\mu \nu}^{e m, N}\left(\mathbf{q}, q_{0}\right)
$$

where $G_{Z}^{\mu \nu} \simeq \eta^{\mu \nu}$ is the $Z$ propagator (the denominator, $1 / M_{Z}^{2}$, has been absorbed in $G_{F}$ ) and $T_{\mu \nu}^{e m, N}\left(\mathbf{q}, q_{0}\right)$ is the Compton scattering amplitude of a virtual $Z$ with momentum $q$ by a nucleon $N$ at rest. In the Born approximation this amplitude reduces to:

$$
\begin{aligned}
T_{\mu \nu}^{Z, N}\left(\mathbf{q}, q_{0}\right)= & \frac{(2 \pi)^{4}}{2} \frac{4 M q^{2}}{q^{4}-4 M^{2} q^{0^{2}}}\left(1+\frac{q^{2}}{2 M^{2}}\right) \\
& \sum_{\text {spin }}\left[\left\langle N\left|J_{\mu}^{e m}(0)\right| N^{\prime}\right\rangle\left\langle N^{\prime}\left|J_{v}^{e m}(0)\right| N\right\rangle+\mu \leftrightarrow v\right]
\end{aligned}
$$

where $M$ is the mass of the nucleon $N$ at rest, $N^{\prime}$ indicates a nucleon with fourmomentum $\left(\mathbf{q}, q_{0}+M\right)$ and the sum is over both its spin states.

In the same approximation, the neutral weak current matrix elements between two nucleons of momenta $p, p^{\prime}=p+q$ and spin $\alpha$ and $\alpha^{\prime}$ can be expressed in the form:

$$
\left\langle N(p, \alpha)\left|J_{\mu}^{e m}(0)\right| N^{\prime}\left(p^{\prime}, \alpha^{\prime}\right)\right\rangle=\bar{u}^{(\alpha)}(p)\left[F_{1}^{N}\left(q^{2}\right) \gamma_{\mu}+i F_{2}^{N}\left(q^{2}\right) \sigma_{\mu v} q^{v}\right] u^{\left(\alpha^{\prime}\right)}\left(p^{\prime}\right)
$$


where $u(p)$ are Dirac spinors and $F_{1}, F_{2}$ are the Dirac and Pauli form factors of the nucleon.

Plugging (4) into (3) and doing a Wick rotation, one can get, after some algebra, the expression for the weak, neutral current induced, nucleon self energy:

$$
\begin{aligned}
\Delta M_{N}^{W-N V}= & -\frac{1}{\pi M^{2}} \int_{0}^{\infty} q d q \int_{0}^{q} d v \sqrt{q^{2}-v^{2}} \frac{4 M q^{2}}{q^{4}+4 M^{2} v^{2}} \\
& {\left[3 q^{2} f_{1}^{Z}\left(q^{2}\right)-\left(q^{2}+2 v^{2}\right) f_{2}^{Z}\left(q^{2}\right)\right] }
\end{aligned}
$$

where the quantities $f_{1}^{Z}\left(q^{2}\right), f_{2}^{Z}\left(q^{2}\right)$ are related to the neutral weak form factors:

$$
\begin{aligned}
f_{1}^{Z}\left(q^{2}\right) & =\frac{\alpha_{W}}{\pi} \frac{\left[G_{M}^{Z}\left(q^{2}\right)\right]^{2}-\left[G_{E}^{Z}\left(q^{2}\right)\right]^{2}}{q^{2}+4 M^{2}} \\
f_{2}^{Z}\left(q^{2}\right) & =\frac{\alpha_{W}}{\pi} \frac{q^{2}\left[G_{M}^{Z}\left(q^{2}\right)\right]^{2}+4 M^{2}\left[G_{E}^{Z}\left(q^{2}\right)\right]^{2}}{q^{2}\left(q^{2}+4 M^{2}\right)}
\end{aligned}
$$

and where

$$
\alpha_{W}=\frac{\sqrt{2} G_{F} M^{2}}{\pi}=0.463 \times 10^{-5}
$$

The sum rule (5) is the contribution to the self mass of the nucleon coming from the isospin-breaking part of the weak interaction which is, as we said above, related to the vector part of the weak neutral current. The weak contribution to the protonneutron mass difference is obtained, then, by straightforward subtraction of the proton and neutron weak neutral vector self masses $\Delta M_{p-n}^{W}=\Delta M_{p}^{W-N V}-\Delta M_{n}^{W-N V}$.

The weak form factors, except for isolated points, have not been measured [16]. However, using CVC, they can be related to the electromagnetic form factors [17]:

$$
\begin{aligned}
G^{p Z} & =\frac{1}{2}\left(G^{p}-G^{n}\right)-2 \sin ^{2} \theta_{W} G^{p}-\frac{1}{2} G^{s Z} \\
G^{n Z} & =-\frac{1}{2}\left(G^{p}-G^{n}\right)-\frac{1}{2} G^{s Z}
\end{aligned}
$$

where we have normalized them to the weak isospin values $G_{E}^{p, n Z}(0)=t_{3 L}$ and where $G^{s}$ is the contribution of the $s$-quark sea to the weak form factor. Measurements show that this latter quantity is very small and we shall neglect it [16].

To compute the $P-N$ mass differences the weak Cottingham formula, we use the "Galster parameterization" $[18,19]$ for the electromagnetic form factors. With these values we obtain the results:

$$
\frac{\left(m^{N}-m^{P}\right)^{W}}{m}=(-5.0 \pm 1.0) \times 10^{-9}
$$

The error was estimated from the known discrepancies of the Galster parameterization with experiment, plus a generous allowance for the largely unknown strange contribution. 
TABLE 1. Upper bounds for the UFF violation parameters.

\begin{tabular}{llc}
\hline$\Gamma$ & $\Delta M^{n-p}=0$ & $\Delta M_{C t}^{n-p}$ \\
\hline$\Gamma^{S}$ & $1.0 \times 10^{-9}$ & $1.1 \times 10^{-9}$ \\
$\Gamma^{E}$ & $1.2 \times 10^{-9}$ & $1.2 \times 10^{-9}$ \\
$\Gamma^{W}$ & $2.8 \times 10^{-2}$ & $1.0 \times 10^{-3}$ \\
\hline
\end{tabular}

Comparison with the results of Eötvös experiments, summarized in references [2, $7,8]$, are shown in table 1 . The first two columns show the upper bounds obtained assuming that a single interaction breaks the equivalence principle. The first column $(\Delta M=0)$ excludes the nucleon structure contribution while the second column $\left(\Delta M_{C t}\right)$ includes it, as computed with the generalized Cottingham formulae. We find that while the inclusion of individual nucleons effect does not change much the upper limit on the strong and electromagnetic violation parameters $\left(10^{-8}\right)$, it lowers the bound on $\Gamma^{W}$ from $\left(3 \times 10^{-2}\right)$ to $\left(\times 10^{-3}\right)$ : an order of magnitude increase in sharpness.

As a final remark, let us observe that while proton-neutron weak mass splitting originates in the neutral currents, the "nuclear" contribution of weak interactions is dominated by the charged ones $[10,11]$. Hopefully, the STEP experiment, with its larger accuracy and better cover of the periodic table may help to put bounds on the separate currents. Even though we should interpret our results with caution, they confirm that Eötvös experiments do test weak interactions effect with an accuracy, at least one order of magnitude, better than previous studies.

\section{REFERENCES}

1. A. Einstein, Ann. Phys. 35, 898 (1911)

2. C. M. Will, Theory and experiment in gravitational physics, Cambridge University Press, Cambridge (1981)

3. S. Weinberg, Gravitation and Cosmology, Wiley, New York (1972)

4. T. Damour and A. M. Polyakov, Nucl. Phys. B 423:532 (1994)

5. J. M. Overduin and P. S. Wesson, Phys. Rep. 283:303 (1997)

6. R. Reinhard, Y. Jafry, and R. Laurence. ESA J., 17:251, 1993.

7. H. Vucetich, Bol. Acad. Nac. Cs. (Córdoba) 61, 1 (1996)

8. T. Damour in Proceedings of the Workshop on the Scientific Applications of Clocks in Space Kluwer (1997).

9. R. Eisberg and R. Resnick, Fisica Cuantica, Limusa, Mexico (1978)

10. M.P. Haughan and C.M. Will, Phys. Rev. Lett. 37:1 (1976)

11. E. Fishbach, M.P. Haughan, D. Tadic and H. Cheng, Phys. Rev. D, 32:154 (1985)

12. W. N. Cottingham, Ann. Phys. N. Y., 25:424 (1963)

13. L. N. Epele, H. Fanchiotti, C. A. García Canal and R. Méndez Galain, in Frontier Phisics: Essays in Honour of Jayme Tiomno S. MacDowell, H. M. Nussenzveig and R. A. Salmeron eds. (1991)

14. H. R. Christiansen, L. N. Epele, H. Fanchiotti and C. A. García Canal, Phys. Lett. B267:164 (1991)

15. R. Méndez Galain La diferencia de masa neutrón-protón Ph. D. Thesis. U. Nac. de La Plata. (1989).

16. U.-G. Meißner Baryon form factors: Model-independent results hep-ph/9907323.

17. N. C. Mukhopadhyay Weak form factors of the nucleon hep-ph/9810039.

18. M. J. Musolf et. al. Phys. Rep. 2391 (1994).

19. S. Galster et al. Nucl. Phys. B 32221 (1971) 